\title{
Transcription factor NFkB regulates the expression of the histone deacetylase SIRT1
}

\author{
Judith Katto ${ }^{1}$, Nicole Engel ${ }^{2}$, Wasim Abbas $^{3}$, Georges Herbein ${ }^{3}$ and Ulrich Mahlknecht ${ }^{1,2,4^{*}}$
}

\begin{abstract}
Background: The NAD-dependent protein deacetylase SIRT1 has a wide range of different targets, which may be regulated either directly through deacetylation and thus potentially altering their activity or localization or indirectly by deacetylation of histones, which in turn alters their transcription rate and availability. SIRT1 is therefore involved in the regulation of many different and fundamental cellular processes such as apoptosis, metabolism, differentiation and cell cycle arrest. It is also involved in the regulation of resistance of cells against oxidative stress and longevity under conditions of caloric restriction. Even though the targets and role of SIRT1 have been studied quite intensively, only little is known about the mechanisms affecting SIRT1 transcriptional regulation. The nuclear factor NFkB is a well-studied and widely known transcription factor, which is involved in the regulation of many important cellular activities. The regulation of NFKB by SIRT1 has been reported recently, but it is, however, still unknown whether a feedback mechanism affects the regulation of SIRT1 too, particularly in view of the fact that putative NFKB binding sites within the SIRT1 promoter suggest just that.
\end{abstract}

Results: In the study presented herein we show that there is activation of the SIRT1 promoter by overexpression of different NFKB subunits. Direct binding of NFKB to the SIRT1 promoter can be demonstrated by an electrophoretic mobility shift assay. Further investigations indicated enhanced expression of SIRT1 on the mRNA levels in cells overexpressing NFKB. A functional assay showed that acetylation of one of the main target proteins of SIRT1 is reduced in these cells.

Conclusions: These finding together indicate SIRT1 expression to be regulated in a positive feedback loop by NFKB. The putative binding sites for NFKB found within the SIRT1 promoter appears to be functional and several NFKB subunits are able to enhance the expression of SIRT1 if they are overexpressed.

Keywords: Protein deacetylase SIRT1, Nuclear factor NF-kappa-B, Apoptosis

\section{Background}

The regulation of activity, cellular localization and stability of nearly all proteins depends on post-transcriptional and/or post-translational modifications. Such modifications are being carried out by a diversity of chemical reactions including (de)phosphorylation, (de)acetylation or ubiquitination and many others. These highly dynamic regulatory processes are controlled and driven by antagonizing enzymes and allow cells to react to environmental

\footnotetext{
* Correspondence: mahlknecht@gmx.de

${ }^{1}$ José Carreras Center for Immunotherapy and Gene Therapy, Saarland University

Medical Center, Kirrbergerstraße Bldg. 45.3, 66421, Homburg/Saar, Germany

${ }^{2}$ Department of Internal Medicine, University of Heidelberg Medical Center,

Im Neuenheimer Feld 410, 69120, Heidelberg, Germany

Full list of author information is available at the end of the article
}

changes and to communicate with each other. Histone deacetylases (HDACs) are one such group of enzymes. HDACs are ubiquitous and catalyze the removal of acetyl groups from both histones and non-histone proteins. The most obvious and best examined role of HDACs is their effect on the constitution and maintenance of chromosomal structure as well as the regulation of global gene expression through modification of the so called histone code and alteration of the accessibility of genes for transcription factors [1]. However, apart from their role as superordinative gene regulators and their role in the control of chromatin structure, some proteins of this family are associated with the distinct regulation of single target proteins, which may specifically be deacetylated [2].

Histone deacetylases are divided into four different classes: three groups of classical HDACs known as class I,

\section{Biomed Central}


class II and class IV HDACs and a fourth class, also being referred to as the 'sirtuins' [3]. The sirtuins differ from the other classes of HDACs based on their dependence on the presence of the coenzyme $\mathrm{NAD}^{+}$[4]. Seven human sirtuins, which are homologs of the yeast silent information regulator 2 (SIR2) protein, have been reported so far. SIR2 is responsible for the transcriptional silencing of silent mating loci, telomeres and ribosomal DNA in yeast. It also suppresses the recombination of rDNA and thereby lowers the formation of toxic extrachromosomal rDNA rings, which extends lifespan in yeast cells dramatically [5]. Yeast SIR2 or mammalian sirtuin overexpression goes along with the global deacetylation of histones [6]. Most of the gene silencing effects that are being associated with sirtuin activity depend on the deacetylation of histone $\mathrm{H} 4$ lysine $16\left(\mathrm{H}_{\mathrm{K} 16}\right)$ [7] and/or histone $\mathrm{H} 3$ lysines 9,14 or 18 $\left(\mathrm{H} 3_{\mathrm{K} 9}, \mathrm{H} 3_{\mathrm{K} 14}, \mathrm{H} 3_{\mathrm{K} 18}\right)$ [8]. Depending on the presence of $\mathrm{NAD}^{+}$, the sirtuins are biological sensors for the cellular energetic and oxidative status. Thus, high levels of NAD ${ }^{+}$ go along with high sirtuins activity [9].

The human deacetylase sirtuin1 (SIRT1) shares the highest degree of homology of all mammalian sirtuins with the yeast protein SIR2. SIRT1 localizes predominantly to the cell nucleus [10] and deacetylates both histones (preferably $\mathrm{H}_{\mathrm{K}_{16}}$ ) and non-histone proteins. SIRT1 antagonizes the histone acetyltransferase p300 [11], which acetylates all histones and some non-histone proteins such as p53 and FOXO3a. SIRT1 is involved in a number of cellular regulation mechanisms affecting cellular lifespan, stress resistance, apoptosis and cell metabolism [12]. The prolongation of cellular lifespan under conditions of caloric restriction has been known for quite some time. It has been described for a number of very different organisms ranging from yeast [13] to nematodes [14] to mammals $[15,16]$ and has been reported to be associated with SIRT1. Caloric restriction causes an increase in the production of $\mathrm{NAD}^{+}$and goes along with increased levels of SIRT1 mRNA $[13,16]$ as well as with increased SIRT1 activity [17]. The positive influence of SIRT1 on longevity in mammals has been reported to depend on increased stress resistance and reduced apoptosis. In addition to the modification of histone proteins, SIRT1 also carries the ability to inactivate a number of non-histone proteins by deacetylation. One of the most prominent examples for this mechanism is the deacetylation of tumor suppressor protein p53. The transcription factor p53 triggers apoptosis in the case of DNA damage or oxidative stress and plays a key role in cellular defense against the development of malignant transformation [18]. The inactivation of p53 by SIRT1 may retard apoptosis and render cells more resistant to oxidative stress $[19,20]$. On the other hand, overexpression of SIRT1 may also promote carcinogenesis because of its inhibitory effect on apoptosis and cell cycle arrest. The forkhead proteins constitute another group of proteins that is also involved in the regulation of apoptosis, stress resistance and metabolism; these proteins are reported to be regulated by SIRT1 [21].

Another protein that may be deacetylated by SIRT1 is the nuclear factor NF-kappa-B (NFKB) [22-24]. NFkB is a ubiquitously distributed transcription factor that controls gene expression of many target genes, thereby affecting important cellular functions such as cell cycle, angiogenesis, adhesion and apoptosis [25]. NFKB is being constituted by homo- and heterodimers of different NFkB/ Rel-proteins, which all share a highly conserved DNA binding/dimerization domain called 'Rel homology ( $\mathrm{RH}$ ) domain' [26]. NFKB/Rel proteins can be divided into two classes. Members of the first class are coded by the genes NFKB1 and NFKB2 both of them normally being transcribed as long isoforms called NFKB-p105 and NFkBp100, which are inactive as long as they have inhibiting $\mathrm{C}$-terminal domains. The shorter active forms of this class, the NFkB-p50 (derived from p105) and NFkB-p52 (derived from p100) are produced by arrested translation or partial proteolysis of the longer forms. The subunit NFkB-p49 is another isoform that can be generated from an alternatively spliced transcript of p100. It is reported to be involved in the activation of the HIV enhancer [27]. The second class contains the proteins c-Rel, RelA (also called NFkB-p65) and RelB, which may activate gene expression in homodimers as well as in heterodimers [28]. The most prevalent and best studied dimer is the heterodimer consisting of the $\mathrm{p} 65$ and p50 protein. NFKB is constitutively transcribed in many different cell types, but it remains inactive because the dimers are bound to inhibitory proteins of the IKB family, which mask their nuclear translocation signal and consequently go along with their localization in the cell cytoplasm. In response to diverse stimuli ІкB proteins are phosphorylated by ІкB kinase (IKK) which triggers their ubiquitination and thus their degradation by the proteasome machinery. $\mathrm{NF \kappa B}$ is subsequently translocated to the nucleus were it interacts with promoters, thus activating the expression of specific target genes [29].

Generally speaking, alterations of SIRT1 gene expression are associated with changes in cell metabolism, differentiation, proliferation and many other fundamental cell features and may therefore affect numerous diseases such as diabetes, cancer or arteriosclerosis. Therefore, regulation of SIRT1 gene expression at the promoter level is of great interest in order to further elucidate our understanding of the molecular mechanisms that involve SIRT1 in both health and disease. The goal of the study presented herein consists in the further elucidation of the interplay of the transcription factor $\mathrm{NF}_{\kappa} \mathrm{B}$, in the reciprocal regulation with SIRT1, taking into consideration possible feedback mechanisms in the regulation of these two proteins. 


\section{Results and discussion}

\section{Human SIRT1 gene promoter analysis}

Alterations in the SIRT1 expression rate are associated with changes in cell metabolism, differentiation, proliferation and many other fundamental cell features and affect many diseases such as diabetes, cancer or arteriosclerosis. But at present little information is available on the molecular mechanisms regulating SIRT1 at the promoter level. In order to further elucidate the regulation of SIRT1 at the promoter level, a search for putative transcription factor binding sites was carried out using the online tool MOTIF Search (http://motif.genome.jp). The search was based on the alignment with the transcription factor database TRANSFAC. Among others, we found the SIRT1 promoter to contain several putative NFKB binding sites. NFKB is a transcription factor that is involved in apoptosis, the regulation of cellular stress, and numerous immunological functions. The NFkB consensus sequence GGGAMTTYCC with $\mathrm{M}$ and $\mathrm{Y}$ being exchangeable bases was found with minor variations in three positions of the SIRT1 promoter. The three mentioned positions are located at the promoter base-pair positions 1,168 to $1,177 \mathrm{bp}, 1,441$ to $1,450 \mathrm{bp}$ and 1,838 to $1,847 \mathrm{bp}$. In accordance with our in silico analyses the latter two $\mathrm{NF}_{k} \mathrm{~B}$ potential binding sites appeared to be specific for the NFkB subunit p65. This suggests that Sirt1 expression could potentially be regulated by $N F \kappa B$.

\section{NFKB regulates the SIRT1 promoter}

In order to further investigate whether $\mathrm{NF} \kappa \mathrm{B}$ was involved in the regulation of SIRT1 at the promoter level, a luciferase reporter plasmid was generated, which contained a 2,228-bp-long fragment of the SIRT1 promoter sequence. This construct was cotransfected in HEK293T cells together with different combinations of expression vectors of the NFKB subunits p49, p50 or p65. Luciferase activity of cell extracts was measured 24 hours after transfection and normalized to the protein content of the cell extracts. Figure 1 plots the results of three independent measurements of luciferase activity relative to the constitutive activity. The constitutive activity was determined by transfection of the reporter plasmid without cotransfection of any expression plasmids for $\mathrm{NF}_{\kappa} \mathrm{B}$. These results show clearly that the Sirt1 promoter is activated in general by overexpression of NFkB. Statistical $P$ values indicated in the diagram were calculated using a Mann-Whitney $U$ Test and show significance for the difference of each $\mathrm{NF}_{\mathrm{K}} \mathrm{B}$ subunit combination versus control $(P \leq 0.05)$. The different combinations of $\mathrm{NF \kappa B}$ subunits we transfected in order to determine the effects of the different homo- and heterodimers of $\mathrm{NF}_{\kappa} \mathrm{B}$ did not show significant differences among each other $(P>0.1)$.

Our experiments showed overexpression of $\mathrm{NF}_{\kappa} \mathrm{B}$ p50 subunit or NFkB p65 subunit alone to increase SIRT1 promoter activity by an average of 8.6 -foldand 7.3 -fold.

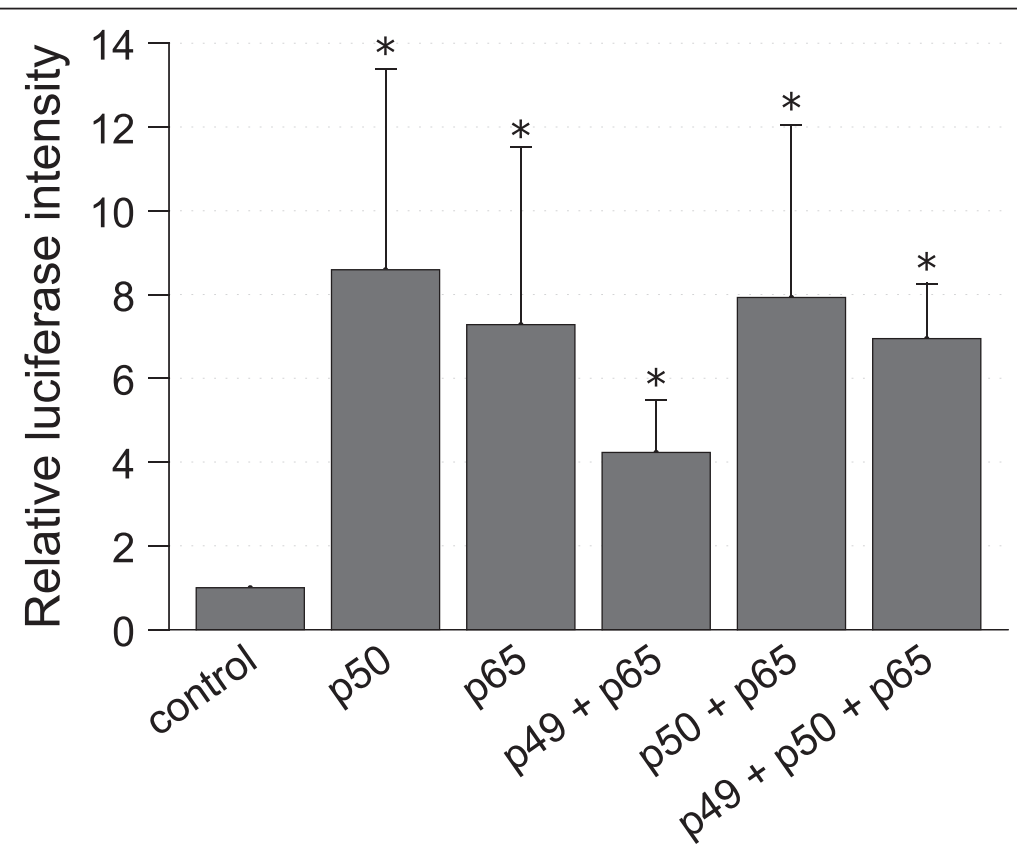

Figure 1 SIRT1-promoter activation after transfection of NFKB subunits. Reporter plasmids containing the SIRT1 promoter were cotransfected with expression vectors for different NFKB subunits in HEK-293 T cells. Luciferase reporter gene assay was done after 24 hours and protein concentration of cell extracts was used to normalize the results. To calculate relative values constitutive luciferase intensity (named control) was measured after transfection of the SIRT1 reporter plasmid without NFkB expression plasmids. $\mathrm{n}=3 .{ }^{*} P \leq 0.05$ versus control group. 
Overexpression of the two NFKB subunits p50 and p65 in combination was identified to have an activating effect on the SIRT1 promoter with an average of about 7.9-fold, which is very similar to the effects of overexpression of the single subunits. The heterodimer p50/p65 represents the most common and best examined variant of $\mathrm{NFKB}$, which makes these results the most interesting. The overexpression of p49 together with p65 also showed SIRT1 promoter activation. The activating effect of this combination seemed to be lower with an average of only 4.2fold, but the difference to the other subunit combinations is not significant. Overexpression of all three subunits together goes along with a seven-fold activation of the SIRT1 promoter, which is similar to the other results. Transfection of the empty reporter plasmid (without Sirt1 promoter) was used as negative control and did not show any luciferase activity.

\section{NFKB regulates SIRT1 expression}

In order to examine whether the effects of promoter activation through overexpression of NFKB in fact had influence on the SIRT1 expression levels, RNA from HEK293T cells was extracted after transient transfection with the $\mathrm{NF} \kappa \mathrm{B}$ expression constructs. In quantitative real-time PCR we observed a 1.8 to 3 -fold upregulation for SIRT1 at the mRNA level (Figure 2) in response to NFkB overexpression for nearly all combinations of NFkB subunits except in the combination of p50 with p65, which had only minor effects on SIRT1 mRNA expression levels in this experiment. The little effect of the overexpression of p50 in combination with p65 on the mRNA level, does not fit with the remarkable promoter activating effects seen for this combination in the luciferase assay. A possible explanation may be based on the fact that the p50/p65 dimer is the most common dimer of NFkB, which has numerous physiological functions in the cell. Therefore, other regulating mechanisms may also be activated that could affect SIRT1 expression.

\section{Functional assay of SIRT1 activity}

A functional assay was carried out not only to investigate the levels of SIRT1 expression but also the effects of NFKB on cell metabolism and other proteins in association with SIRT1. In order to prove relevant changes in SIRT1 activity after NFKB overexpression we investigated the acetylation status of histone $\mathrm{H} 4$ lysine residue $16\left(\mathrm{H} 4_{\mathrm{K} 16}\right)$, which is targeted and deacetylated by SIRT1. We isolated the histone fraction of cells after transfection with defined NFkB subunits and analyzed the histone $\mathrm{H} 4$ acetylation status by western blot technique. Figure $3 \mathrm{~A}$ shows a remarkable decrease of acetylated $\mathrm{H} 4_{\mathrm{K} 16}$ in cells overexpressing NFKB. For quantification of the results, blots were scanned and acetylated histone $\mathrm{H} 4$ was normalized to the amount of total histone $\mathrm{H} 4$ on the same blot. To be sure, that the effects are not due to the circumstances of cell culture or transfection in general, which may change acetylation status of histones because it induces lots of metabolical reactions and stress, we compared the results of cells transfected with $\mathrm{NFKB}_{\mathrm{K}}$ vector with those transfected with an empty vector. Figure 3B

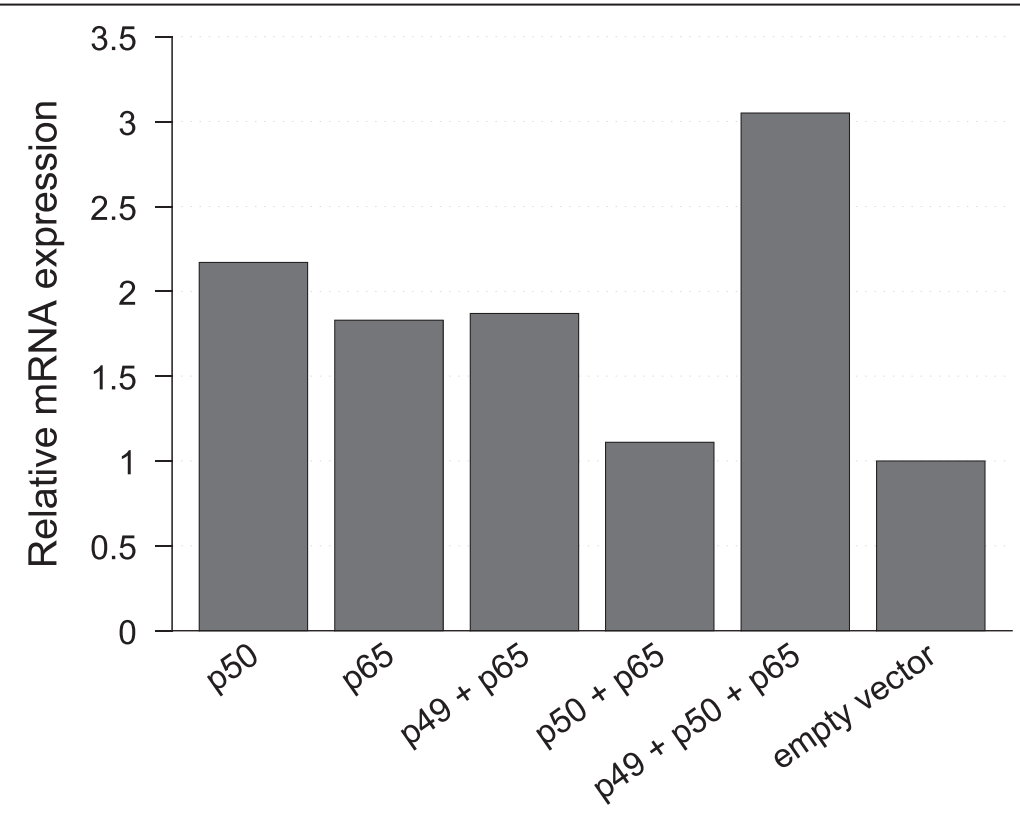

Figure 2 SIRT1 expression on mRNA level. HEK-293 T cells were transfected with expression plasmids of different NFkB subunits and harvested 24 hours later. Cells transfected with empty vector were used as reference for calculation of relative values. The mRNA levels of Sirt1 gene were measured by real-time-PCR. 

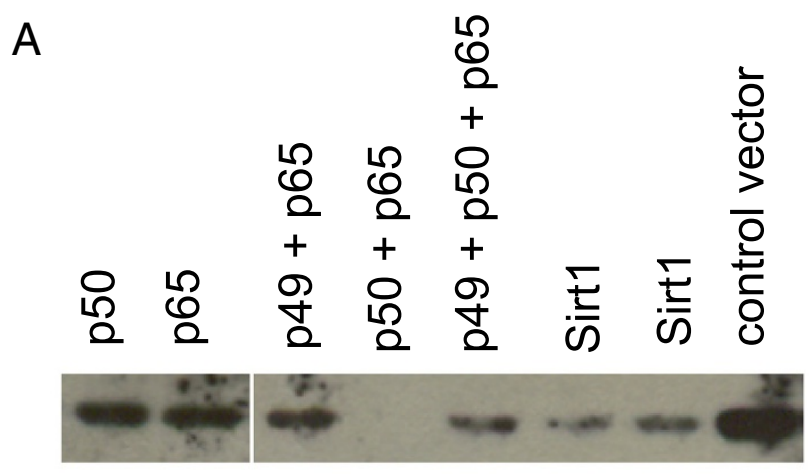

H4K16
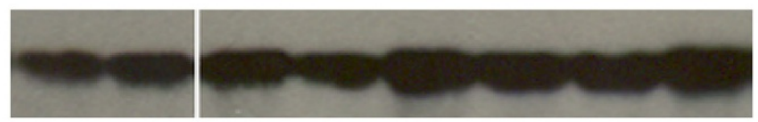

total $\mathrm{H} 4$

B

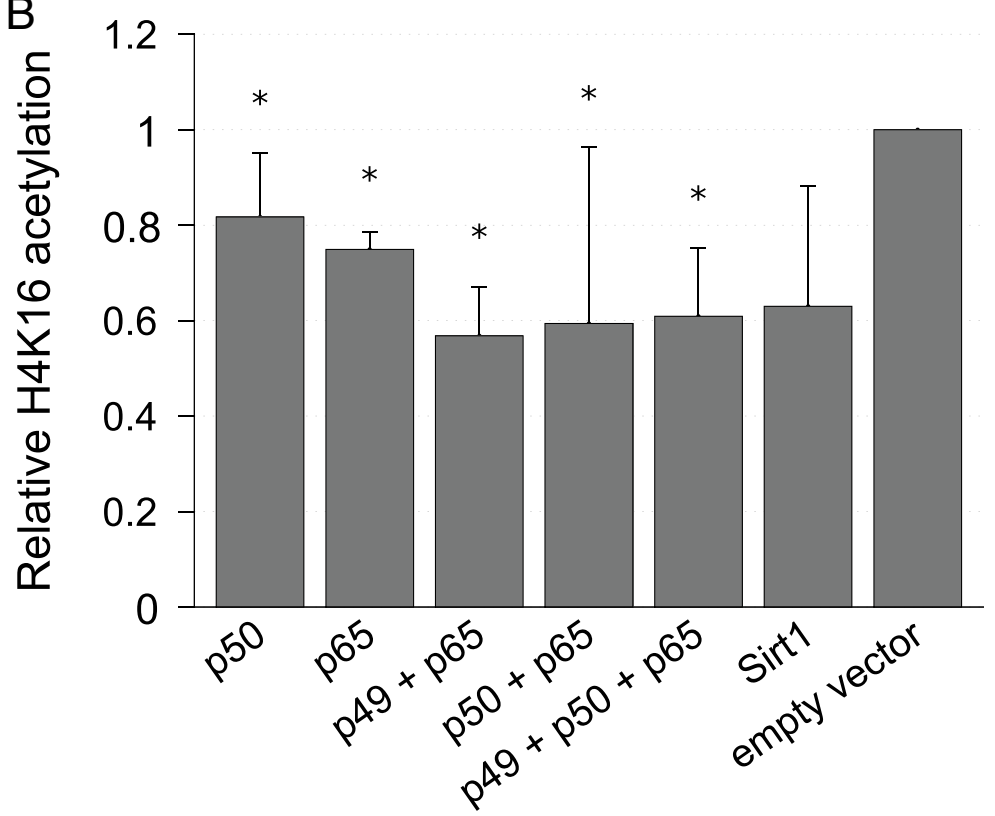

Figure 3 Histone acetylation status in response to SIRT1 activity. Cells were transfected with expression vectors for different NFKB subunits. Expression vector for SIRT1 was used as a positive control. Histones were isolated after 24 hours and acetylation status was assessed by western blot. Transfection with empty vector was used as a reference control. (A) Detection was carried out with an antibody specific for acetylated histone $\mathrm{H} 4_{\mathrm{K} 16} \mathrm{and}$ with another antibody binding to histone $\mathrm{H} 4$ independently of the acetylation status (total $\mathrm{H} 4$ ). (B) Detection films of blots were scanned and quantified in silico. Values of $\mathrm{H}_{\mathrm{K} 16}$ were normalized to total $\mathrm{H} 4$ and fold changes relative to control were calculated. $\mathrm{n}=3$. ${ }^{*} P>0.05$ versus empty vector.

shows that the overexpression of NFkB subunits in all combinations resulted in a significant reduction of the relative amount of acetylated histone $\mathrm{H} 4$. The amount of reduction in cells overexpressing two or more $\mathrm{NFkB}$ subunits was similar to the reduction caused by direct overexpression of SIRT1. In cells overexpressing only one subunit of NFKB protein the effects are less but still detectable. These results suggest that overexpression of NFKB has activating effects on the deacetylation of histone $\mathrm{H} 4$, which could be mediated through induction of SIRT1 expression, particularly when two or three of the NFkB subunits are overexpressed, thus allowing for a formation of heterodimers.

\section{Binding of NFKB to SIRT1 promoter}

To investigate the role of different putative NFKB binding sites, truncations of the SIRT1 promoter were generated (Figure 4). The 2,228 bp and 1,148 bp SIRT1 promoter fragments contained all three putative $\mathrm{NF}_{\mathrm{K}} \mathrm{B}$ binding sites; fragments with length ranging from 1,026 bp, to $921 \mathrm{bp}$ and $887 \mathrm{bp}$ in length contained only two NFkB binding sites and the $668 \mathrm{bp}$ fragment contained only one NFKB binding site.

An electrophoretic mobility shift assay was done to measure the binding of $\mathrm{NFKB}$ to the promoter and to investigate the relation of the three potential binding sites. In order to induce $\mathrm{NF}_{\kappa} \mathrm{B}$ expression, cells were treated 


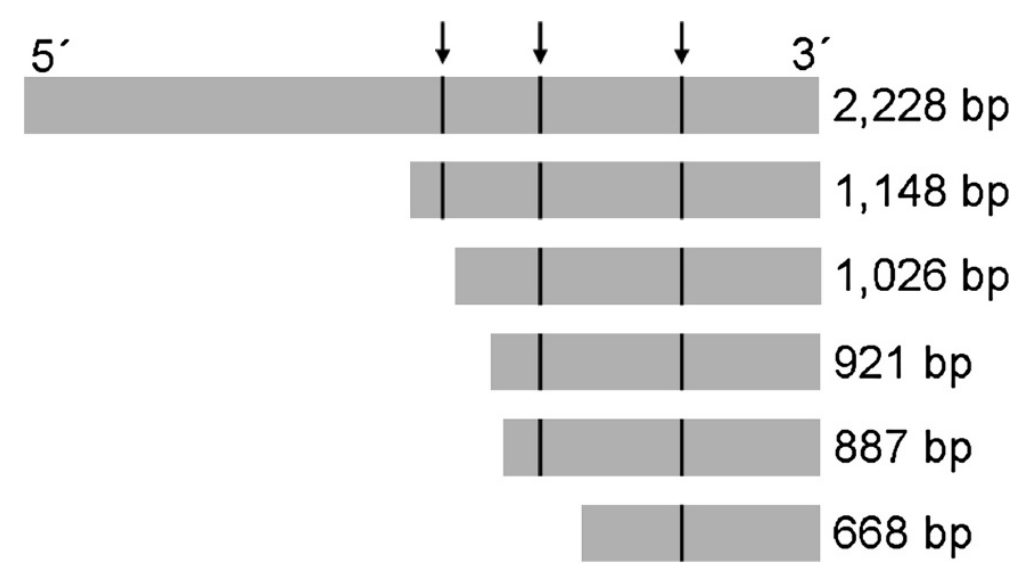

Figure 4 Sirt1 promoter truncations. Schematic illustration of Sirt1 promoter fragments used for electrophoretic mobility shift assay. The positions of NFkB binding sequences are marked by arrows.

with TNF $\alpha$. Nuclear extracts from both treated and untreated cells were isolated and incubated with biotin labeled DNA fragments. The reaction mixture was resolved on a $6 \%$ non-denaturing polyacrylamide gel. For all of the tested promoter fragments signals were detected (Figure 5), which proves binding of NFKB. The putative binding site contained in the short fragment is likely to be involved in the NFKB binding of the promoter, but because all of the fragments showed a signal, it is not possible to decide whether the binding is mediated solely by this single site or whether all three sites are involved. Nuclear extracts from untreated cells were used as negative controls. $\mathrm{NF} \kappa \mathrm{B}$ oligonucleotides were used as a positive control to prove induction of NFkB expression by TNF $\alpha$ and to compare the position of the bands on the blot. The positive control showed a signal that was much stronger than the labeled promoter fragments. This may be caused by the high sensitivity of the control, which also could explain the signal seen for the NFKB oligonucleotides when incubated with untreated cells.

\section{Conclusions}

$\mathrm{NFkB}$ is a transcription factor that can be found in nearly all cell types. NFKB regulates immune reactions and is involved in the regulation of cellular stress resistance and apoptosis. Also, it has recently been shown that the NFKB binding sequence is a gene regulatory motif that is most essentially involved in the regulation of cellular aging. SIRT1 is a highly conserved protein involved in many cellular processes in both health and disease. It has been reported to be involved in the extension of cellular life span under conditions of caloric restriction in many species. Its positive influence on stress resistance of cells is widely known and its activation prevents apoptosis and cell cycle arrest. Since both proteins, SIRT1 as well as $\mathrm{NF} \kappa \mathrm{B}$, are involved in the regulation of immune response, cellular stress, and aging, a regulatory connection between them would not really be very surprising. In some studies SIRT1 has been shown to interact with NFkB and to repress its activity by deacetylation [22,24]. But at present little information is available on the molecular mechanisms regulating the expression of SIRT1at the promoter level. In the study presented herein, we show that in addition to the already known regulation of $\mathrm{NFkB}$ by SIRT1, NFאB overexpression enhances SIRT1 promoter activity. Increased SIRT1 enzymatic activity caused by NFKB overexpression was shown in a functional assay. The direct binding of the transcription factor $\mathrm{NFKB}$ to the Sirt1

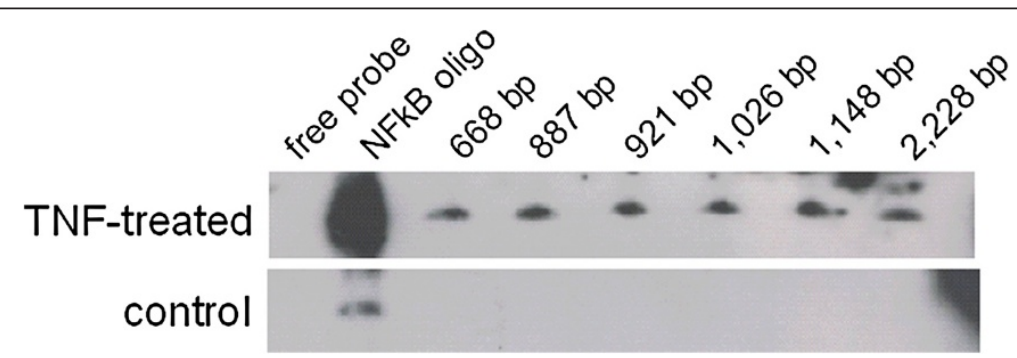

Figure 5 Binding of NFKB to DNA fragments of truncated SIRT1 promoter. U937 cells were treated with TNFa to induce NFKB expression. Nuclear extracts of TNFa-treated and untreated control cells were incubated with different biotin labeled DNA fragments of the SIRT1 promoter and were analyzed by electrophoretic mobility shift assay. An NFkB oligonucleotide was used as the positive control. 
promoter was demonstrated by electrophoretic mobility shift assays. With these results a reciprocal feedback regulation of SIRT1 at the promoter level through transcription factor $N_{k} B$ is shown to take place.

The conserved $\mathrm{NF}_{\kappa} \mathrm{B}$ binding motif can be found three times within the human SIRT1 promoter sequence. Distinguishing which of the three potential NFKB binding sites was responsible for the activation of the promoter could not be made since all of the created SIRT1 promoter truncations showed binding of NFKB. With reporter plasmids containing the human SIRT1 promoter sequence and luciferase assays, we were able to show that high expression levels of NFKB subunits were able to specifically activate SIRT1 at the promoter level. Our experiments show that the $N_{F} B$ binding motifs within the SIRT1 promoter are functional, and that $\mathrm{NF}_{\kappa} \mathrm{B}$ can act as a positive regulator of SIRT1 at the promoter level. In order to further examine whether $\mathrm{NF}$ KB overexpression on SIRT1 promoter activation caused a real increase in SIRT1 expression, we performed RT-PCR experiments using cells overexpressing $\mathrm{NF}_{k} \mathrm{~B}$ that showed elevated levels of SIRT1 mRNA for most of the used NFKB subunits. As a functional assay to measure SIRT1 activity the acetylation status of histone $\mathrm{H} 4_{\mathrm{K} 16}$, which is one of the most common targets of the deacetylase SIRT1, was examined. The decrease in acetylation of $\mathrm{H} 4_{\mathrm{K} 16}$ under conditions of $\mathrm{NF} \mathrm{B}$ overexpression indicates not only that SIRT1 protein levels are increased, but also that this has measurable effects on downstream targets. This means that at least some of the signaling cascades and pathways that activate $N F \kappa B$ may influence the expression of SIRT1 and the acetylation status of its target proteins. The involvement of both of these proteins in the development and progression of numerous diseases such as cancer, arteriosclerosis or diabetes renders this regulation mechanism an interesting target for further investigations with regard to the development of novel molecular therapeutics affecting SIRT1 regulation. SIRT1 is one of the most interesting targets for potential epigenetic therapeutics and the ongoing attempts to create specific activators or deactivators for SIRT1 may benefit from better knowledge about its regulation by intercellular transcription factors. Taken together, these findings provide new insights into the regulation of SIRT1 and interactions between NFkB and SIRT1. They may promote a better understanding of the mechanism involved in regulation of proteins that control procedures as important as metabolism and aging.

\section{Methods}

\section{Antibodies and plasmids}

Anti-histone H4 monoclonal antibody (anti-H4) was purchased from Abcam (Cambridge, UK) and antibody specific for Histone $\mathrm{H} 4$ acetylated at lysine 16 (anti-H4 $4_{\mathrm{K} 16}$ ) was purchased from Upstate via Biomol, (Hamburg, Germany). Secondary antibodies (anti-rabbit and anti-mouse horseradish peroxidase conjugates) were purchased from Santa Cruz Biotechnology (Santa Cruz, California, USA).

NFKB expression plasmids for subunits p49, p50 and p65 were based on pRSV expression vector systems and were provided by the National Institutes of Health (NIH) AIDS Research and Reference Reagent Program. For construction of luciferase reporter plasmids, human SIRT1 promoter from genomic DNA was amplified by PCR. The amplified region spanned the entire SIRT1 promoter region. The promoter was inserted into the luciferase reporter plasmid pGL2-basic, which was purchased from Promega (Mannheim, Germany). All plasmids were propagated using competent DH5 $\alpha$ E. coli and were isolated with a Nucleobond PC100 Kit from Macherey \& Nagel (Düren, Germany). All transfection experiments were carried out in HEK293T cells.

\section{Luciferase reporter gene assays}

HEK293T-cells were cultured in 12-well plates with DMEM supplemented with 10\% FCS and were cotransfected with the luciferase SIRT1 promoter or empty pGL2basic vector constructs and different combinations of expression vectors encoding NFKB subunits. In order to normalize the total amount of DNA that was used per transfection, empty vector was added in order to adjust the total amount of DNA. Transfection was done using the nanoparticle-based transfection reagent Nanofectin (PAA, Pasching, Austria) and medium was changed 2 hours after transfection. Transfection efficiency was assessed by UV-microscopy after transfection of green lantern constructs (Promega, Mannheim, Germany). Cells were harvested after 24 hours and luciferase activity was measured using the luciferase reporter assay system (Promega, Mannheim, Germany) in accordance with the manufacturer's instructions. The activity of each sample was normalized to its protein concentration measured on a UV-Vis spectrophotometer Nanodrop ${ }^{\text {тM }}$ ND1000 (PEQLAB, Erlangen, Germany) using DC Protein assay (BioRad, Hercules, California, USA) based on the Lowry method. Relative activity was calculated as fold difference in relation to the control vector pGL2basic. Luciferase assays were performed in duplicates and every experiment was repeated at least twice.

\section{Quantitative real-time PCR}

Total RNA from HEK293T cells was extracted with the Trifast reagent (Peqlab, Erlangen, Germany) in accordance with the manufacturer's instructions. Total RNA was treated with Turbo DNAse (Life Technologies, Carlsbad, USA) and was reverse transcribed with the High capacity cDNA Reverse Transcription Kit from Applied Biosystems (Darmstadt, Germany). The cDNA samples were amplified in quantitative real-time PCR using a Light Cycler 
2.0 system and DNA Master SYBR Green Kit both from Roche (Penzberg, Germany). The primers that were used for amplification of human SIRT1 were as follows:

forward primer 5' - ACGCTGGAACAGGTTGCGGG A - 3',

reverse primer $5^{\prime}$ - AAGCGGTTCATCAGCTGGGCA C - 3'.

Thermal reaction cycles were as follows: initial cycle at $95^{\circ} \mathrm{C}$ for $10 \mathrm{~min}$, which was then followed by 45 cycles at $95^{\circ} \mathrm{C}$ for $10 \mathrm{sec}, 60^{\circ} \mathrm{C}$ for $4 \mathrm{sec}$ and $72^{\circ} \mathrm{C}$ for $16 \mathrm{sec}$. GAPDH was used as an internal control to normalize measured $\mathrm{Ct}$ values of each sample, and data were analyzed using the delta-delta $\mathrm{Ct}$ method.

\section{Extraction of histones and western blot}

HEK293T cells were cultured in 12-well plates and were harvested with $100 \mu \mathrm{l}$ lysis buffer after incubation for 24 hours. While cells were being mixed vigorously, $25 \mu \mathrm{l}$ of $2 \mathrm{M}$ sulphuric acid were added drop by drop, and cells were then placed on ice for $1 \mathrm{~h}$. After centrifugation at $13,000 \mathrm{rpm}$, supernatants were collected, mixed with $400 \mu \mathrm{l}$ of $20 \%$ trichloroacetic acid and incubated on ice for histone precipitation. After $1 \mathrm{~h}$ the mixture was spun down and the pellets containing histones were washed twice with acidified acetone $(0.1 \% \mathrm{HCl})$ and once with pure acetone prior to resuspension in water. Extracted histones were separated on a $15 \%$ polyacrylamide gel and blotted onto a nitrocellulose membrane according to the full-wet western blot technique. Acetylated histones were detected with anti $\mathrm{H}_{\mathrm{K} 16}$ antibody. For detection, the ECL plus western blotting detection reagent from GE Healthcare (Little Chalfont, UK) was used. After that, an antibody binding to $\mathrm{H} 4$ independent of the acetylation status was used in order to detect total histone H4. Blots were scanned and quantifications were calculated with the ImageQuant5.1 software (GE Healthcare, Little Chalfont, UK). Signal of acetylated $\mathrm{H} 4$ was normalized to the signal of total $\mathrm{H} 4$ in order to eliminate effects depending on the western blot technique itself.

\section{Table 1 Enzymes for digestion of SIRT1} promoter truncations

\begin{tabular}{ll}
\hline Fragment length & Enzymes for plasmid digestion \\
\hline $2,228 \mathrm{bp}$ & Hind3 \\
$1,148 \mathrm{bp}$ & Bgl2 and Hind3 \\
$1,026 \mathrm{bp}$ & $\mathrm{Kpn} 1$ and Hind3 \\
$921 \mathrm{bp}$ & $\mathrm{Sma1}$ \\
$887 \mathrm{bp}$ & $\mathrm{Bg} / 2$ and Hind3 \\
$668 \mathrm{bp}$ & Bgl2 and Hind3 \\
\hline
\end{tabular}

\section{Promoter truncation and electrophoretic mobility shift assay (EMSA)}

The generation of the different promoter fragments was done with different methods. The fragment with $921 \mathrm{bp}$ was generated by exonuclease digestion. Fragments with $1,148 \mathrm{bp}$ and 1,026 bp were generated through restriction enzyme digestion and subsequent re-ligation. Fragments with $887 \mathrm{bp}$ and 668 bp were amplified by PCR from genomic DNA and inserted into an empty vector. Plasmids were transformed into E. coli DH5a for propagation and extracted accordingly. A total of $2 \mu \mathrm{g}$ plasmid DNA were digested with restriction enzymes (Table 1) and then run on a $0.8 \%$ agarose gel. Digested fragments from each construct were cut out while the gel was monitored under UV control, and DNA elution was done with a Silica Beads DNA gel extraction kit (Fermentas, Waltham, USA). DNA from each construct was labeled with biotin using the Biotin 3' End DNA Labeling Kit (Pierce Biotechnology, Waltham, USA). In order to stimulate $\mathrm{NF \kappa B}$ expression, U937 cells were treated with recombinant TNF $\alpha$ at a concentration of $10 \mathrm{ng} / \mathrm{ml}$ for 30 minutes. Control cells were left untreated. Nuclear extracts $(10 \mu \mathrm{g})$ were then incubated with a specific biotinlabeled DNA sample before electrophoretic mobility shift assay (EMSA) was done using a Light Shift Chemiluminescent EMSA kit (Pierce Biotechnology, Waltham, USA) in accordance with the manufacturer's instructions.

\section{Abbreviations}

NFKB: Nuclear factor of kappa light polypeptide gene enhancer in B-cells; SIRT1: Sirtuin 1.

\section{Competing interests}

The authors declare that they have no competing interests.

\section{Authors' contributions}

UM, JK, NE, and WA conceived and designed the experiments. JK, NE, and WA analyzed the data. JK wrote the first draft of the manuscript. UM contributed to the writing of the manuscript. UM GH, WA, and NE made critical revisions. All authors reviewed and approved the final manuscript.

\section{Acknowledgements}

This work was supported by institutional funds from the Saarland University, grants from the University of Franche-Comté (UFC) and the Region Franche-Comté (RECH-FON12-000013) to G.H. W.A. is a recipient of a doctoral scholarship from the Higher Education Commission, Pakistan. We thank Diasorin SA and Roche for their financial support.

\section{Author details}

1José Carreras Center for Immunotherapy and Gene Therapy, Saarland University Medical Center, Kirrbergerstraße Bldg. 45.3, 66421, Homburg/Saar, Germany. ${ }^{2}$ Department of Internal Medicine, University of Heidelberg Medical Center, Im Neuenheimer Feld 410, 69120, Heidelberg, Germany. ' Department of Virology, Pathogens \& Inflammation Research Unit UPRES EA4266, SFR FED 4234, University of Franche-Comté, CHU Besançon, Besançon, France. ${ }^{4}$ Department of Haematology/Oncology, St. Lukas Klinik Solingen, Schwanenstraße 132, 42697, Solingen, Germany. 


\section{References}

1. Yang XJ, Seto E: The Rpd3/Hda1 family of lysine deacetylases: from bacteria and yeast to mice and men. Nat Rev Mol Cell Biol 2008, 9:206-218.

2. Haberland M, Montgomery RL, Olson EN: The many roles of histone deacetylases in development and physiology: implications for disease and therapy. Nat Rev Genet 2009, 10:32-42.

3. Marks PA, Miller T, Richon VM: Histone deacetylases. Curr Opin Pharmacol 2003, 3:344-351.

4. Landry J, Slama JT, Sternglanz R: Role of NAD(+) in the deacetylase activity of the SIR2-like proteins. Biochem Biophys Res Commun 2000, 278:685-690.

5. Kaeberlein M, McVey M, Guarente L: The SIR2/3/4 complex and SIR2 alone promote longevity in Saccharomyces cerevisiae by two different mechanisms. Genes Dev 1999, 13:2570-2580.

6. Imai S, Armstrong CM, Kaeberlein M, Guarente L: Transcriptional silencing and longevity protein $\mathrm{Sir} 2$ is an NAD-dependent histone deacetylase. Nature 2000, 403:795-800.

7. Vaquero A, Scher M, Lee D, Erdjument-Bromage H, Tempst P, Reinberg D: Human SirT1 interacts with histone $\mathrm{H} 1$ and promotes formation of facultative heterochromatin. Mol Cell 2004, 16:93-105.

8. Feige JN, Auwerx J: Transcriptional targets of sirtuins in the coordination of mammalian physiology. Curr Opin Cell Biol 2008, 20:303-309.

9. Rajendran R, Garva R, Krstic-Demonacos M, Demonacos C: Sirtuins: molecular traffic lights in the crossroad of oxidative stress, chromatin remodeling, and transcription. J Biomed Biotechnol 2011, 2011:368276.

10. Michishita E, Park JY, Burneskis JM, Barrett JC, Horikawa I: Evolutionarily conserved and nonconserved cellular localizations and functions of human SIRT proteins. Mol Biol Cell 2005, 16:4623-4635.

11. Bouras T, Fu M, Sauve AA, Wang F, Quong AA, Perkins ND, Hay RT, Gu W, Pestell RG: SIRT1 deacetylation and repression of p300 involves lysine residues 1020/1024 within the cell cycle regulatory domain 1. J Biol Chem 2005, 280:10264-10276.

12. Michan S, Sinclair D: Sirtuins in mammals: insights into their biological function. Biochem J 2007, 404:1-13.

13. Lin SJ, Defossez PA, Guarente L: Requirement of NAD and SIR2 for lifespan extension by calorie restriction in Saccharomyces cerevisiae. Science 2000, 289:2126-2128.

14. Tissenbaum HA, Guarente L: Increased dosage of a sir-2 gene extends lifespan in Caenorhabditis elegans. Nature 2001, 410:227-230.

15. Boily G, Seifert EL, Bevilacqua L, He XH, Sabourin G, Estey C, Moffat C, Crawford S, Saliba S, Jardine K, Xuan J, Evans M, Harper ME, McBurney MW: SirT1 regulates energy metabolism and response to caloric restriction in mice. PLoS One 2008, 3:e1759.

16. Cohen HY, Miller C, Bitterman KJ, Wall NR, Hekking B, Kessler B, Howitz KT, Gorospe M, de Cabo R, Sinclair DA: Calorie restriction promotes mammalian cell survival by inducing the SIRT1 deacetylase. Science 2004, 305:390-392.

17. Bordone L, Guarente L: Calorie restriction, SIRT1 and metabolism: understanding longevity. Nat Rev Mol Cell Biol 2005, 6:298-305.

18. Levine AJ: p53, the cellular gatekeeper for growth and division. Cell 1997, 88:323-331.

19. Vaziri H, Dessain SK: Ng Eaton E, Imai Sl, Frye RA, Pandita TK, Guarente L, Weinberg RA: hSIR2(SIRT1) functions as an NAD-dependent p53 deacetylase. Cell 2001, 107:149-159.

20. Luo J, Nikolaev AY, Imai S, Chen D, Su F, Shiloh A, Guarente L, Gu W: Negative control of p53 by Sir2alpha promotes cell survival under stress. Cell 2001, 107:137-148.

21. Van Der Heide LP, Hoekman MF, Smidt MP: The ins and outs of FoxO shuttling: mechanisms of FoxO translocation and transcriptional regulation. Biochem J 2004, 380:297-309.

22. Salminen A, Kauppinen A, Suuronen T, Kaarniranta K: SIRT1 longevity factor suppresses NF-kappaB -driven immune responses: regulation of aging via NF-kappaB acetylation? BioEssays 2008, 30:939-942.

23. Salminen A, Ojala J, Huuskonen J, Kauppinen A, Suuronen T, Kaarniranta K: Interaction of aging-associated signaling cascades: inhibition of NFkappaB signaling by longevity factors FoxOs and SIRT1. Cell Mol Life Sci 2008, 65:1049-1058

24. Yeung F, Hoberg JE, Ramsey CS, Keller MD, Jones DR, Frye RA, Mayo MW: Modulation of NF-kappaB-dependent transcription and cell survival by the SIRT1 deacetylase. EMBO J 2004, 23:2369-2380.

25. Baldwin AS: Control of oncogenesis and cancer therapy resistance by the transcription factor NF-kappaB. J Clin Invest 2001, 107:241-246.
26. Hayden MS, Ghosh S: Signaling to NF-kappaB. Genes Dev 2004, 18:2195-2224.

27. Schmid RM, Perkins ND, Duckett CS, Andrews PC, Nabel GJ: Cloning of an NF-kappa B subunit which stimulates HIV transcription in synergy with p65. Nature 1991, 352:733-736.

28. Siggers T, Chang AB, Teixeira A, Wong D, Williams KJ, Ahmed B, Ragoussis J, Udalova IA, Smale ST, Bulyk ML: Principles of dimer-specific gene regulation revealed by a comprehensive characterization of NF-kappaB family DNA binding. Nat Immunol 2012, 13:95-102.

29. Karin $M$, Ben-Neriah Y: Phosphorylation meets ubiquitination: the control of NF-[kappa]B activity. Annu Rev Immunol 2000, 18:621-663.

doi:10.1186/1868-7083-5-11

Cite this article as: Katto et al:: Transcription factor NFKB regulates the expression of the histone deacetylase SIRT1. Clinical Epigenetics 2013 5:11.

\section{Submit your next manuscript to BioMed Central and take full advantage of:}

- Convenient online submission

- Thorough peer review

- No space constraints or color figure charges

- Immediate publication on acceptance

- Inclusion in PubMed, CAS, Scopus and Google Scholar

- Research which is freely available for redistribution

Submit your manuscript at www.biomedcentral.com/submit
C Biomed Central 